\title{
Carcinoma ex pleomorphic adenoma of the sublingual gland: a case report
}

\author{
Yasunori Ariyoshi ${ }^{1}$, Masashi Shimahara ${ }^{1}$, Toshiyuki Konda ${ }^{1}$ and Motomu Tsuji ${ }^{2}$ \\ We report a case of carcinoma ex pleomorphic adenoma of a sublingual gland in a 70-year-old man. Under a clinical diagnosis of benign \\ salivary gland tumor, excision of the mass with the sublingual salivary gland in an en bloc fashion via an intraoral approach was \\ performed. Histopathologically, there was a rupture of the fibrous capsule and diffuse cell-rich sheets composed of myoepithelial cells \\ with round nuclei were also seen. Immunohistochemically, the cells that composed of cell rich sheets were positive to smooth muscle \\ actin. Final diagnosis of myoepithelial carcinoma ex pleomorphic adenoma was made.
}

International Journal of Oral Science (2012) 4, 50-53; doi:10.1038/ijos.2012.3; published online 12 January 2012

Keywords: Pleomorphic adenoma; malignant; myoepithelial carcinoma; sublingual gland; magnetic resonance imaging

\section{INTRODUCTION}

Salivary gland tumors occurring in the sublingual gland are rare as compared to other locations, such as the parotid and submandibular glands. Among malignant salivary gland tumors, the most frequent type is adenoid cystic carcinoma, followed by adenocarcinoma and carcinoma ex pleomorphic adenoma. ${ }^{1}$ The malignant component is frequently a poorly differentiated carcinoma, high-grade adenocarcinoma or salivary duct carcinoma. However, most other types of salivary gland carcinomas have been described in carcinoma ex pleomorphic adenoma, and some cases show diverse differentiation with several distinct types within the tumor mass. ${ }^{2}$ According to the World Health Organization histological classification published in 2005, malignat changes in the pleomorphic adenoma include three different types: carcinoma ex pleomorphic adenoma, carcinosarcoma and metastasizing pleomorphic adenoma. The macroscopic features that suggest malignant transformation in pleomorphic adenoma include poorly defined and/or infiltrative tumor margins, the presence of foci of hemorrhage, and necrosis. Also the coexistent benign and malignant elements are considered as well. ${ }^{3}$

Herein, we report a case of carcinoma ex pleomorphic adenoma that occurred in the sublingual gland, and presented histological findings of microinvasion beyond the original capsule and abundant myoepithelial cells.

\section{CASE REPORT}

A 70-year-old Japanese man was referred to our clinic for further evaluation of a mass located in the left floor of the mouth. There were no subjective symptoms, including pain and tenderness, and the patient had not noticed it until his dentist pointed it out. Extraoral examination results were unremarkable and no abnormal mass in the face or lymphadenopathy in the bilateral neck was seen. An intraoral examination revealed a painless, non-tender, elastic hard, smooth mass measuring $1.5 \mathrm{~cm} \times 1.5 \mathrm{~cm}$. The mass was covered by intact oral mucosa and it was not fixed to the surrounding structures, including the mandible (Figure 1). A reduced secretion of saliva from the left orifice of Wharton's duct was found. In a magnetic resonance (MR) examination, a space occupying mass lesion, which showed homogeneous low signal intensity on T1-weighted images and homogeneous intermediate signal intensity on T2-weighted images, was delineated (Figure 2). On dynamic MR images, tumor located in sublingual space was gradually enhanced as compared to that of surrounding sublingual glands (Figure 3 ). The mass was located in the sublingual space

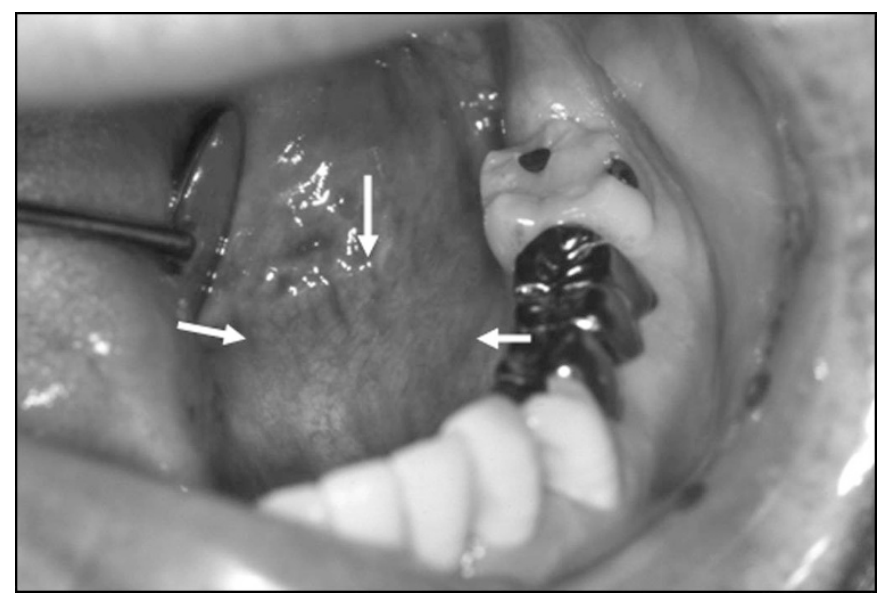

Figure 1 Intraoral findings. A physical examination found a painless, nontender, elastic hard, smooth mass that was covered by intact oral mucosa and not fixed to the mandible. Arrows: tumor 

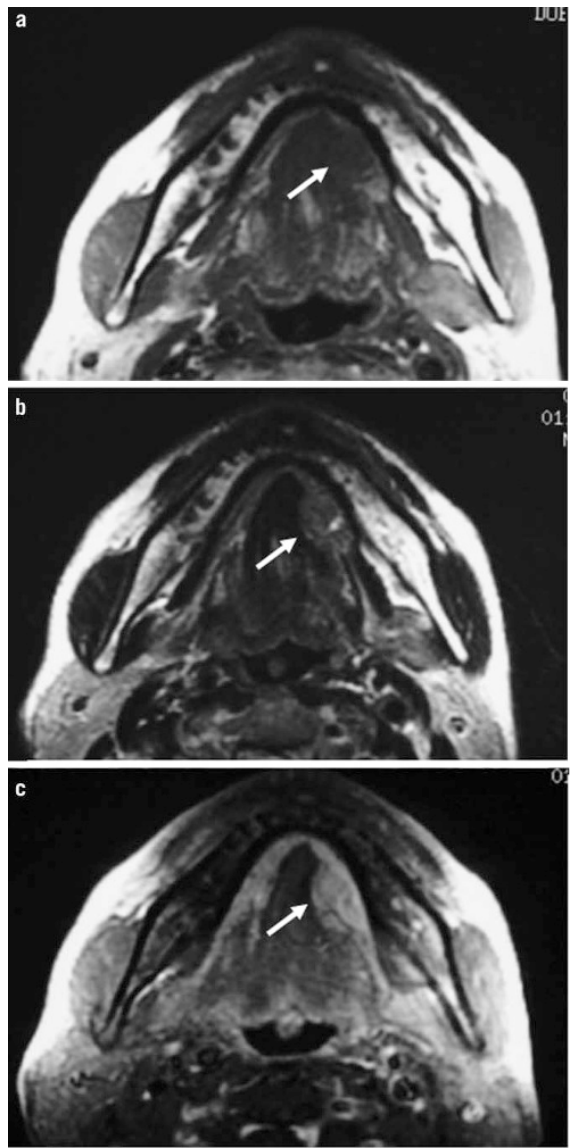

Figure 2 MR images. MR images showing a well-circumscribed mass lesion located in the right sublingual space. Arrows: tumor. (a) T1 weighted image. (b) T2 weighted image. (c) Enhanced T1 weighted image. and a clear interface with the sublingual gland was evident. We made a clinical diagnosis of a benign salivary gland tumor originating from the sublingual salivary gland.

Since tumors arising from the sublingual gland have a high chance of being malignant, ${ }^{4}$ excision of the mass with the sublingual salivary gland in an en bloc fashion via an intraoral approach was performed under general anesthesia. During the operation, the tumor mass was found located deep within the sublingual gland and above the mylohyoid muscle, and a sharp and blunt dissection from the mandible, extrinsic tongue musculature, and mylohyoid muscle was performed. Wharton's duct was also resected, while the lingual nerve could be preserved. The postoperative course was uneventful, and there was no recurrence or metastasis for 5 years and 10 months postsurgically.

The macroscopic appearance of the resected specimen was solid in nature and it was completely encapsulated. The cut surface showed a grayish white, homogeneous, solid mass with no bleeding foci or necrotic areas (Figure 4). Histopathological examination revealed that the tumor was composed of a pleomorphic adenoma consisting of myxoid, chondroid and mucoid materials, as well as duct-like structures and cell-rich mesenchymal tissues (Figure 5a). Although it was well encapsulated, there was a rupture of the fibrous capsule and tumor cells had slightly invaded the surrounding fat tissues (Figure 5b). The surgical margins were free of tumor with abundant safety margin. Diffuse cell-rich sheets composed of myoepithelial cells with round nuclei were also seen, among which there were mitotic figures and atypical cells (Figure $5 \mathrm{c}$ and $5 \mathrm{~d}$ ). There were no sarcomatous components found within the tumor.

Immunohistochemical examinations, including smooth muscle actin, S-100 protein and glial fibrillary acidic protein (GFAP), cytokeratin were performed. In the parenchyma, S-100 protein and GFAP-positive cells were found scattered. Staining for cytokeratin highlighted the ductal cells, whereas stromal cells that had differentiated into myxoid, chondroid and mucoid cells were not stained. The
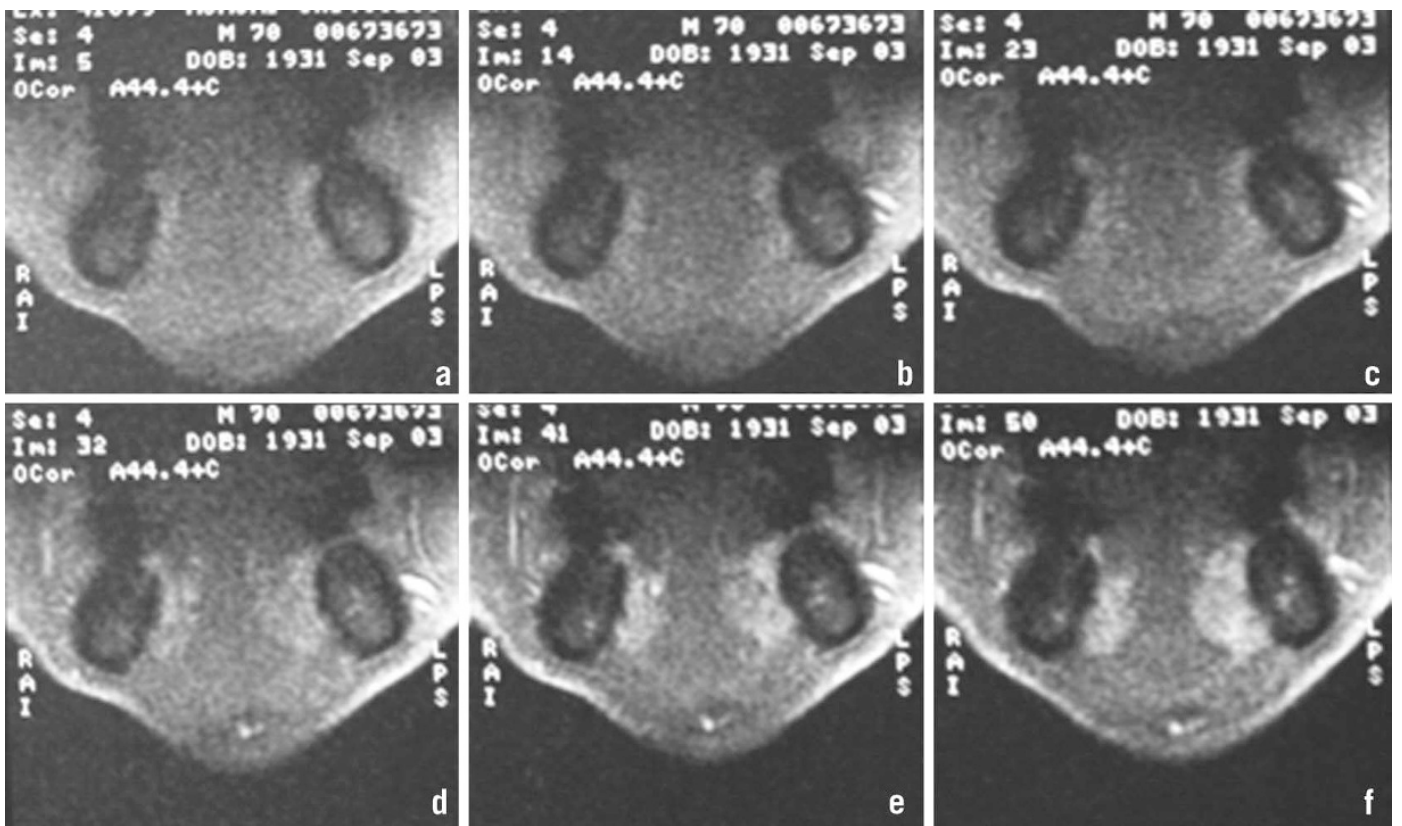

Figure 3 Fat suppressed dynamic enhanced MR images. Fat suppressed dynamic enhanced MR images showing gradually enhanced mass lesion located in sublingual space. (a) Precontrast image. (b-e) Early phase images. (f) Delayed image. 

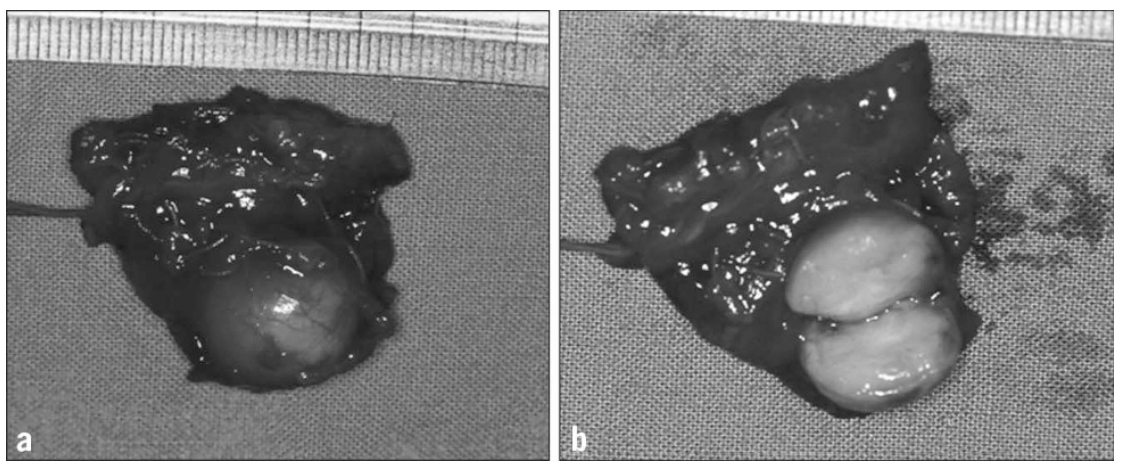

Figure 4 The cut surface of the resected specimen showed a grayish white, homogeneous, and solid mass without bleeding foci or necrotic areas. (a) Gross appearance. (b) Cut slice of the resected specimen.
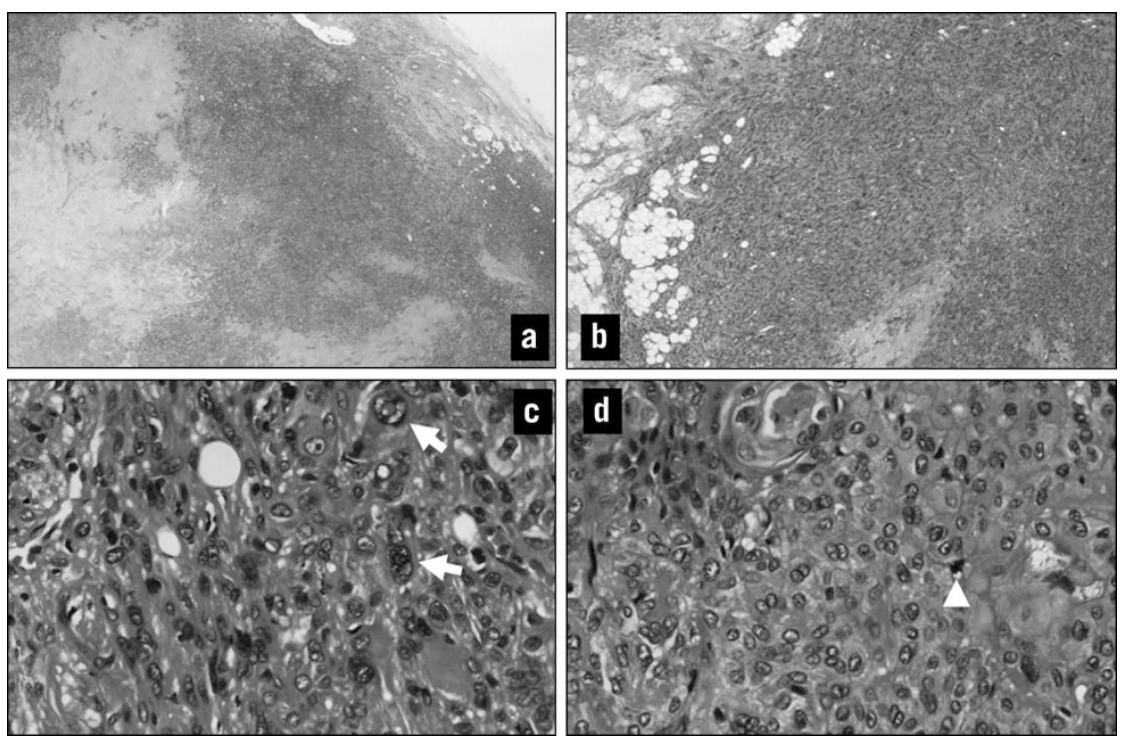

Figure 5 Histopathological features of the resected specimen. (a) The tumor was composed of a pleomorphic adenoma that consisted of myxoid, chondroid, and mucoid materials. (b) There was a rupture of the fibrous capsule and tumor cells had slightly invaded the surrounding fat tissues. (c, arrows) Diffuse cell-rich sheets composed of myoepithelial cells with round nuclei were seen, among which there were atypical cells and (d, arrowhead) mitotic figures. Hematoxylin and eosin staining; magnification: $a, \times 6.6 . b, \times 13.2 . c, d \times 132$.
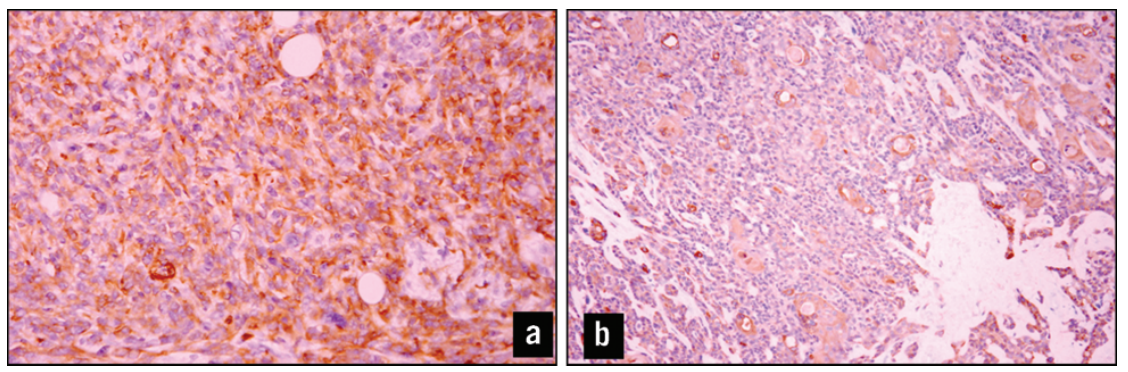

Figure 6 Immunohistochemical staining of the resected specimen. $(\mathbf{a}, \times 66)$ Tumor cells expressed reactivity with antibodies to SMA and $(\mathbf{b}, \times 66)$ duct like cells were positive to cytokeratin.

cells that composed of cell-rich sheets were positive to smooth muscle actin (Figure 6). Postoperative ultrasound examination of the neck and ${ }^{18} \mathrm{~F}$-fluorodeoxyglucose positron emission tomography study revealed no metastatic deposits. Based on these findings, a final diagnosis of myoepithelial carcinoma ex pleomorphic adenoma (pT1NOM0) was made. Additional treatments including neck dissection and adjuvant radiation were not performed, because there were no evidences of recurrence and metastasis, and patient adapted a 'waitand-see' approach.

\section{DISCUSSION}

Salivary gland tumors are rarely found located in the sublingual gland, ${ }^{5-6}$ though such masses have been shown to be malignant in $80 \%-90 \%$ of the reported cases. ${ }^{7}$ Nagler and Laufer ${ }^{8}$ reported that they 
did not observe any benign tumors occurring in the sublingual glands. Clinically, an asymptomatic swelling in the floor of the mouth is the most common complaint associated with malignant sublingual gland tumors, though one case was incidentally discovered by a dentist, ${ }^{9}$ which was the same as with the present patient, who did not notice the mass until pointed out by his dentist. Tumors of the sublingual salivary gland are generally not recognized until they reach an advanced stage, mainly because of minimal symptomatology. ${ }^{10}$ In order to detect a sublingual malignant neoplasm in the early stage, it is necessary for dentists to notice such sublingual masses. In addition, it is important to rule out malignancy when a sublingual mass is presented.

A diagnosis of malignant salivary neoplasm must be considered for every patient who has a swelling in the area of the major salivary glands or a submucosal mass in the oral cavity or pharynx, even if the swelling has been present for years. ${ }^{1}$ In the present case, there were no apparent features that indicated a malignant tumor in the results of our physical examination or in diagnostic images. Clinically, we routinely use magnetic resonance imaging (MRI) as a diagnostic tool for mass lesions located in soft tissues. ${ }^{11}$ Sumi et al. ${ }^{12}$ reported MR findings for an adenoid cystic carcinoma originating from the sublingual gland and a gingival squamous cell carcinoma that had invaded the sublingual gland, which both demonstrated destruction of the gland. In the present case, the tumor brought displacement rather than destruction of the sublingual gland. Asaumi et al. ${ }^{13}$ reported that dynamic MRI was useful in differentiating malignant from benign tumors, as well as for detecting the extent of invasion of a sublingual carcinoma. According to the marginal appearance and enhancement timing of the present mass lesion, we considered that it was benign rather than malignant in nature. Further, since a large part of the tumor margin was encapsulated, we thought it was difficult to diagnose the present case as malignant in nature, because of the marginal features on diagnostic images, including those obtained with MRI.

In the World Health Organization classification of salivary gland tumors, ${ }^{3}$ the histological proportion of benign versus malignant components can be quite variable. Occasionally, extensive sampling is necessary to find the benign component and in rare case, a benign remnant might not be found. As for infiltrative growth, three subtypes can be distinguished: non-invasive, minimally invasive and invasive. In the present case, our final diagnosis was minimally invasive carcinoma ex pleomorphic adenoma, as there was microinvasion beyond the capsule.

Tortoledo et al. ${ }^{14}$ reported a histopathologic subclassification for 37 cases of carcinomas ex pleomorphic adenoma, which yielded 13 cductal, 10 undifferentiated, 9 terminal duct and 3 myoepithelial types, with two cases that could not be classified because of the small csize of the surgical specimen. In the present case, because cells showing myoepithelial differentiation were abundant, it could be subclassified as a myoepithelial type. Tortoledo et al. ${ }^{14}$ also reported two variables, measured invasion in millimeters and histological subclassification cof the malignant neoplasm, as valuable guides for prognosis and biologic behavior. However, they found no definite association between gross dimensions of the tumor and incidence of recurrence or metastasis.

Carcinoma ex pleomorphic adenoma of the sublingual gland is extremely rare and frequently misdiagnosed. When recurrence and distant metastasis occur, survival is so low; therefore, early and adequate removal of that carcinoma ex pleomorphic adenoma is extremely critical. Although metastasis to the lung is rare, patients treated for carcinoma ex pleomorphic adenoma should be investigated for distant metastasis, such as to the lungs and bone, ${ }^{15}$ thus long term follow-up examinations for local recurrence and systemic metastasis of our patient are essential.

1 Andersen LJ, Therkildsen $\mathrm{MH}$, Ockelmann $\mathrm{HH}$ et al. Malignant epithelial tumors in the minor salivary glands, the submandibular gland, and sublingual gland. Cancer 1991, 68(1): 2431-2437.

2 Eveson JW. Malignant neoplasms of the salivary gland. In: Tompson LD (ed.) Head and Neck Pathology. Philadelphia: Churchill Livingstone, 2006: 321-370.

3 Gnepp DR, Brandwein-Gensler MS, El-Naggar AK et al. Carcinoma ex pleomorphic adenoma. In: Barnes L, Eveson JW, Reichart P, Sidransky D (eds.) World Health Organization Classification of Tumours. Pathology and Genetics of Head and Neck Tumours. Lyon: IARC Press, 2005: 242-243.

4 Fletcher CD. Diagnostic Histopathology of Tumors. London: Churchill Livingstone, 2000: 233-303.

5 Spiro RH. Salivary neoplasms: overview of a 35-year experience with 2807 patients. Head Neck Surg 1986; 8(3): 177-184

6 Satko I, Stanko P, Longaueroia I. Salivary gland tumours treated in the stomatological clinics in Bratislava. J Cranio-Maxillofac Surg 2000; 28(1): 56-61.

7 Eneroth CM. Incidence and prognosis of salivary-gland tumors at different histologic sites. Acta Otolaryngol 1970; 263: 174-178.

8 Nagler RM, Laufer D. Tumors of the major salivary glands: review of 25 years of experience. Anticancer Res 1997; 17(1B): 701-708.

9 Spiro RH. Treating tumors of the sublingual glands, including a useful technique for repair of the floor of the mouth after resection. Am J Surg 1995; 170(5): 457460.

10 Rinaldo A, Shaha AR, Pellitteri PK et al. Management of malignant sublingual salivary gland tumors. Oral Oncol 2004; 40(1): 2-5.

11 Ariyoshi $Y$, Shimahara M. Magnetic resonance imaging of a submental dermoid cyst report of a case. J Oral Maxillofac Surg 2003; 61(4): 507-510.

12 Sumi M, Izumi M, Yonetsu $\mathrm{K}$ et al. Sublingual gland: MR features of normal and diseased states. AJR Am J Roentogenol 1999; 172(3): 717-722.

13 Asaumi J, Shigehara $\mathrm{H}$, Konouchi $\mathrm{H}$ et al. Assessment of carcinoma in the sublingual region based on magnetic resonance imaging. Oncol Rep 2002; 9(6): 1283-1287.

14 Tortoledo ME, Luna MA, Batsakis JG. Carcinomas ex pleomorphic adenoma and malignant mixed tumors. Histopathological Indexes. Arch Otolaryngol 1984; 110(3): 172-176.

15 Akan H, Yildiz L, Unal R. Carcinoma ex pleomorphic adenoma of the minor salivary gland with pulmonary metastasis. Diagn Interv Radiol 2008; 14(1): 3-5.

This work is licensed under a Creative Commons SOMERIGHSHESERVED Attribution-NonCommercial-NoDerivative Works 3.0
Unported License. To view a copy of this license, visit http:// creativecommons.org/licenses/by-nc-nd/3.0 